\title{
Joint Surgery in Tunisian Rheumatoid Arthritis Patients: Prevalence and Risk Factors
}

\author{
Olfa SAIDANE ${ }^{1}$, Leila GAFSI ${ }^{2}$, Aicha Ben TEKAYA ${ }^{1}$, Ines MAHMOUD ${ }^{1}$, \\ Rawdha TEKAYA ${ }^{1} \mathbb{D}$, Leila ABDELMOULA ${ }^{1} \mathbb{D}$ \\ ${ }^{1}$ Department of Rheumatology, Charles Nicolle Hospital, Tunis, Tunisia \\ ${ }^{2}$ Department of Rheumatology, Polyclinic El Omrane, Tunis, Tunisia
}

\begin{abstract}
Objectives: This study aims to assess the prevalence of joint surgery in Tunisian patients with rheumatoid arthritis (RA) and to determine the risk factors of surgical treatment.

Patients and methods: This retrospective cross-sectional study was performed over a period of 15 years between January 2000 and December 2014 and included 500 Tunisian patients with RA (78 males, 422 females; mean age 53.4 years; range, 21 to 83 years). The prevalence of joint surgery indication was evaluated. Clinical, paraclinical and therapeutic characteristics of RA were compared according to the need of surgery.

Results: Female to male ratio was 5 . The indication of joint surgery was noted in 59 patients (12\%). Knee joint surgery was the most performed surgical procedure (56\% of surgical treatment). A decrease in surgery prevalence from $30 \%$ in 2004 to $4 \%$ in 2013 was noted. Statistical study showed that factors associated with joint surgery were: delayed diagnosis $(p=0.037)$, long RA duration $(p=0.017)$, young onset of RA ( $p<0.001)$, presence of joint deformities $(p=0.034)$, presence of osteoporosis $(p=0.029)$, presence of antinuclear antibodies $(p<0.001)$, combination therapy of methotrexate with other conventional synthetic disease-modifying anti-rheumatic drugs (csDMARDs) $(p=0.001)$, short period of first medical treatment ( $p=0.012)$ and high erythrocyte sedimentation rate (ESR) $(p=0.027)$. In multivariate analysis, three factors were independently related to the use of joint surgery: age at disease onset [odds ratio (OR): $2.79995 \%$ confidence interval $(\mathrm{Cl}): 1.49-5.22 ; \mathrm{p}=0.01$ ], high ESR level $(\mathrm{OR}: 2.80795 \% \mathrm{Cl}: 1.5-5.24 ; \mathrm{p}=0.01)$ and association of methotrexate with other csDMARDs (OR: $3.50095 \% \mathrm{Cl}: 1.61-7.56 ; \mathrm{p}=0.01$ ).

Conclusion: Twelve percent of RA patients needed joint surgical treatment. Predictive factors of surgery were age at disease onset, high ESR level and association of methotrexate with other csDMARDs.

Keywords: Joints, rheumatoid arthritis, risk factors, surgery, Tunisian.
\end{abstract}

\begin{abstract}
Rheumatoid arthritis (RA) is a chronic inflammatory disease characterized by polyarticular synovial inflammation and progressive joint destruction. ${ }^{1}$ Its management has evolved markedly over recent years due to early diagnosis and the development of new treatments. ${ }^{2,3}$ Orthopedic surgery is an integral part of the treatment of RA that is mainly reserved for severe and advanced forms with
\end{abstract}

failure of medical treatment. The indication for surgical treatment has been modified over recent years by better management of RA. ${ }^{4-6}$

In addition to its role in restoring joint function, orthopedic surgery was shown to be effective in reducing pain and improving quality of life. ${ }^{5}$ Its place in the management of RA remains to be defined, particularly with the change in the management strategy and the use of biological

Received: March 06, 2019 Accepted: October 05, 2019 Published online: December 02, 2019

Correspondence: Leila Gafsi, MD. Department of Rheumatology, Charles Nicolle Hospital, 1006 Tunis, Tunisia. Tel: +21671896144 e-mail: gafsileila@yahoo.fr 
treatments. ${ }^{6}$ Moreover, risk factors of joint surgery should be evaluated for a better disease management.

Few data relating to joint surgery and its evolution in recent years exist in the literature. Therefore, in this study, we aimed to assess the prevalence of joint surgery in Tunisian patients with RA and to determine the risk factors of surgical treatment.

\section{PATIENTS AND METHODS}

This retrospective cross-sectional study was performed at Charles Nicolle Hospital of Tunis over a period of 15 years between January 2000 and December 2014 and included 500 patients with RA (78 males, 422 females; mean age 53.4 years; range, 21 to 83 years) responding to the American College of Rheumatology (ACR) 1987 and/or ACR/ European League Against Rheumatism (EULAR) 2010 criteria.,8 Data were collected from files of patients who were hospitalized in the rheumatology department. Patients with RA history of less than two years, patients whose surgery was indicated for etiology not directly related to RA (fracture, osteonecrosis, osteochondritis, etc.) and patients with incomplete data were excluded. The study protocol was approved by the Charles Nicolle Hospital of Tunis Ethics Committee. A written informed consent was obtained from each patient. The study was conducted in accordance with the principles of the Declaration of Helsinki.

Socio-demographic features including age, sex, and living environment were collected. The existence of comorbidities such as diabetes mellitus, hypertension, cardiovascular, renal disease and smoking history was determined. Different disease characteristics were collected including age at disease onset, time to disease diagnosis and disease duration. The presence of extra-articular manifestations and articular deformities was noted. Weight and height were specified and body mass index (BMI) was calculated. Based on the World Health Organization definitions, patients were subsequently categorized as underweight (BMI <18.5), normal (BMI 18.5-24.99), overweight (BMI 25-29.99) and obese (BMI $\geq 30$ ).
Laboratory data were collected including the erythrocyte sedimentation rate (ESR) and C-reactive protein (CRP). Disease activity parameters including painful joints number, swelling joints number and the 28-joint disease activity score (DAS28) (ESR and CRP) ${ }^{9}$ were determined. Assessment of disease impact on patients' general state and their quality of life was evaluated by the Health Assessment Questionnaire (HAQ). ${ }^{10}$

Immunologic investigations including rheumatoid factor (RF), anti-cyclic citrullinated peptide (anti-CCP) antibody and antinuclear antibodies (ANAs) were determined.

Radiological assessment allowed searching for possible joint space narrowing and erosions on X-rays of the hands and feet, atlantoaxial instability on the X-ray of the cervical spine and hip involvement on the X-ray of the pelvis. RA was considered erosive if erosions were present on standard radiographs. Kellgren and Lawrence classification system in arthrosis was used to determine the importance of articular destructions. The presence of osteoporosis was also determined based on densitometry.

Concerning medical treatment, the medications received including conventional synthetic disease-modifying anti-rheumatic DRUGS (csDMARDS) and/or biologic disease modifying anti-rheumatic DRUGS (bDMARDS) were also documented.

Concerning surgical treatment, all cases of surgical indication were recorded and different parameters regarding surgery were collected: the indication of surgery, type of surgical procedure, number of surgery and time between RA diagnosis and surgery. Variation in prevalence of surgical treatment indication over the years was determined.

\section{Statistical analysis}

The IBM SPSS version 20.0 for Windows software was used for statistical analysis (IBM Corp., Armonk, NY, USA). The Chi square test was used to study the relationship between two qualitative characters and the Student's t-test for the comparison of quantitative characters. Statistical significance was accepted for a $p$ value $<0.05$. 
Patients were divided into two groups: group 1; patients with surgical treatment indication and/or who underwent surgery and group 2; patients without surgical treatment indication. Analytic study was based on the comparison of these two groups. The evaluation of risk factors for surgery was calculated by the odds ratio (OR).

In order to identify the risk factors related to surgical treatment independently, we conducted a multivariate analysis and adjusted ORs were calculated to measure the proper role of each factor.

\section{RESULTS}

Socio-demographic and clinical characteristics of the patients were summarized in Table 1 . Parameters concerning height and weight were available in 205 patients (41\%). The mean BMI was $28.1 \pm 5.4 \mathrm{~kg} / \mathrm{m}^{2}$ ranging from 15.80 to $40.32 \mathrm{~kg} / \mathrm{m}^{2}$, while 139 patients (27.2\%) were overweighed or obese. The HAQ was evaluated only in 102 patients. Mean HAQ was $1.6 \pm 0.8$ ranging from 0.2 to 3 .

Table 1. Socio-demographic and clinical characteristics of patients

\begin{tabular}{|c|c|c|c|}
\hline \multirow[b]{2}{*}{ Variables } & \multicolumn{3}{|c|}{ Results } \\
\hline & $\mathrm{n}$ & $\%$ & Mean \pm SD \\
\hline Age (year) & & & $53.4 \pm 12.0$ \\
\hline Sex & & & \\
\hline Female & 422 & 84.4 & \\
\hline Living in urban zone & 405 & 81 & \\
\hline Smoking history & 55 & 11 & \\
\hline Comorbid illness & 125 & 25 & \\
\hline Age at disease onset (year) & & & $41.3 \pm 13.1$ \\
\hline Disease duration (year) & & & $12.1 \pm 8.0$ \\
\hline Time to diagnosis (year) & & & $5.4 \pm 6.6$ \\
\hline Extra-articular manifestations & 312 & 62.4 & \\
\hline Painful joints number & & & $10.5 \pm 8.6$ \\
\hline Swelling joints number & & & $4.8 \pm 4.9$ \\
\hline DAS28-ESR & & & $5.9 \pm 1.4$ \\
\hline DAS28-CRP & & & $5.2 \pm 1.3$ \\
\hline Erosive character & 450 & 90 & \\
\hline Articular deformities & 415 & 83 & \\
\hline Osteoporosis & 120 & 24 & \\
\hline
\end{tabular}

Concerning biologic investigations, ESR was increased in $97.4 \%$ of males and $89.2 \%$ of females, while CRP level was increased ( $>6 \mathrm{mg} / \mathrm{L}$ ) in $79.4 \%$ of patients. RF measured in 445 patients was positive in $71.4 \%(n=357)$. Anti-CCP was evaluated only in $17 \%$ of patients and it was positive in $60 \%(n=51)$.

Concerning treatment, 429 patients (85.7\%) received anti-inflammatory medications while 407 patients $(81.4 \%)$ received corticosteroid. All patients had at least one csDMARD. Methotrexate was received by 386 patients (77.2\%), 197 patients (39.4\%) received sulfasalazine, and 62 patients (12.4\%) leflunomide. A combination of methotrexate and sulfasalazine was prescribed in 50 patients (10\%). Only 282 patients (56.4\%) maintained the first treatment prescribed for a period > six months.

Biologic DMARDs were prescribed in 135 patients (27\%): 35 patients (7\%) received rituximab and 100 patients (20\%) received tumor necrosis factor alpha (TNF- $\alpha$ ) inhibitors.

In standard radiographies, RA was erosive in 450 patients (90\%). Uni- or bilateral hip involvement was noted in 65 patients (13\%). Atlantoaxial instability was noted in 67 patients (13.4\%).

Surgical treatment was indicated by orthopedists in 59 RA patients (11.8\%). Among these 59 patients, four patients refused surgery. Concerning the 55 patients who underwent surgery, 50 had one surgical procedure, four had two surgical procedures and one patient had four surgical procedures. All patients who had surgical treatment were in stage 4 of Kellgren and Lawrence classification.

A total of 62 surgical acts were practiced. Knee joint arthroplasty was the most performed

Table 2. Different surgical procedures practiced in rheumatoid arthritis patients

\begin{tabular}{lcc}
\hline Types of surgical procedures & Surgery number & Surgery (\%) \\
\hline Total knee replacement & 35 & 56 \\
Total hip replacement & 14 & 23 \\
C1-C2 arthrodesis & 8 & 13 \\
Synovectomy & 3 & 5 \\
Ulnar head resection & 2 & 3 \\
Total & 62 & 100
\end{tabular}




\begin{tabular}{|c|c|c|c|c|c|}
\hline & \multicolumn{2}{|c|}{$\begin{array}{l}\text { Group 1: Surgical treatment } \\
\qquad(\mathrm{n}=59)\end{array}$} & \multicolumn{2}{|c|}{$\begin{array}{l}\text { Group 2: No surgical treatment } \\
\qquad(\mathrm{n}=441)\end{array}$} & \multirow[b]{2}{*}{$p$} \\
\hline & $\mathrm{n}$ & $\%$ & $\mathrm{n}$ & $\%$ & \\
\hline Age (year) & & 51.10 & & 53.73 & 0.536 \\
\hline Sex-ratio & & 0.18 & & 0.13 & 0.927 \\
\hline Living in urban zone & 41 & 69.5 & 304 & 68.9 & 0.753 \\
\hline Smoking history & 10 & 16.9 & 42 & 9 & 0.656 \\
\hline Comorbid illness & 18 & 30.5 & 155 & 35.1 & 0.467 \\
\hline Age at disease onset (year) & & 35.36 & & 42.12 & $<0.001$ \\
\hline Disease duration (year) & & 15.75 & & 11.6 & 0.017 \\
\hline Time to diagnosis (year) & & 7.58 & & 5.13 & 0.037 \\
\hline Painful joints number & & 9.86 & & 10.46 & 0.524 \\
\hline Swelling joints number & & 7.82 & & 4.87 & 0.950 \\
\hline $\mathrm{ESR}(\mathrm{mm} / \mathrm{h})$ & & 77.36 & & 65.94 & 0.027 \\
\hline $\mathrm{CRP}(\mathrm{mg} / \mathrm{L})$ & & 35.63 & & 31.67 & 0.444 \\
\hline DAS28-ESR & & 5.9 & & 5.91 & 0.972 \\
\hline DAS28-CRP & & 5.37 & & 5.23 & 0.597 \\
\hline Erosive character & & 95 & & 89 & 0.177 \\
\hline Articular deformities & & 95 & & 82 & 0.034 \\
\hline Use of methotrexate & 47 & 79.6 & 339 & 76.8 & 0.619 \\
\hline Use of salazopyrine & 17 & 28.8 & 180 & 40.8 & 0.089 \\
\hline Use of leflunomide & 5 & 8.4 & 57 & & 0.325 \\
\hline $\begin{array}{l}\text { Association methotrexate and other } \\
\text { csDMARD }\end{array}$ & & 22 & & 8.3 & 0.001 \\
\hline Use of bDMARD & & 22 & & 27 & 0.350 \\
\hline
\end{tabular}

surgical procedure. Surgical acts were summarized in Table 2. The rate of joint surgery treatment was 12 to $16 \%$ between 2000 and 2003. A maximum prevalence reaching 30\% was noted in 2004 . A decrease in surgical indications was observed between 2005 and 2013 with a rate of 4\% noticed during the year 2013.

During the study period, eight acts of C1-C2 arthrodesis were recorded, of which two were performed in 2006. Only three acts of synovectomy were recorded during the year 2014. Tow acts of resection of the ulnar head were performed in 2002 and 2004 and this act has not been practiced since 2005. The average time between the first signs of RA and joint surgery was $7.0 \pm 7.0$ years.

An analytic study was conducted to compare the two groups (group 1; patients with surgical treatment indication and/or who underwent surgery and group 2; patients without surgical treatment indication). Results of the analytic study were summarized in Table 3.

Statistical study showed that the BMI seemed not to have any impact on the indication of surgery $(p=0.059)$. Additionally, no significant relationship was found between HAQ index and surgical treatment in our study (1.58 vs. 1.85 ; $\mathrm{p}=0.120)$.

The comparison between the two groups according to immunologic findings did not reveal any difference concerning $\mathrm{RF}$ and anti-CCP ( $83 \%$ vs. $80 \% ; p=0.574$ and $69 \%$ vs. $62 \%$; $\mathrm{p}=0.275$, respectively) whereas a significant difference was noted concerning ANA levels (23\% vs. $6 \% ; p=<0.001)$.

Concerning treatment, there was no significant association between the initial csDMARD 
prescribed and the use of joint surgery in our study. The molecule used in first intention did not appear to influence the surgical indication $(p=0.081)$. Moreover, patients who had higher maintenance rate of the first molecule of csDMARD had lower risk of joint surgery $(p=0.012)$.

A significant statistical relationship was found between the use of methotrexate in association with another csDMARD and the frequency of joint surgery $(p=0.001)$. The number of patients receiving bDMARD was lower in group 1 without any statistical significance $(p=0.350)$.

The statistical analysis revealed that the rate of surgical treatment decreased from 30\% in 2004 to $4 \%$ in 2013. An increase in the use of methotrexate and biological treatments was concomitant with a reduction in joint surgery indications over time. Between 2005 and 2013 , the reduction in orthopedic surgery was concomitant with the introduction of biological treatments.

The analytic study showed that the factors statistically associated with joint surgery were: delayed diagnosis (OR: 1.9 95\% confidence interval [CI]: 1.1-3.5), long RA duration (OR: 2.9 95\% CI: 1.1-3.6), young onset of RA (OR: 2.9 95\% CI: 1.6-5), presence of joint deformities (OR: 4.160 95\% CI: 1-13.1), presence of osteoporosis (OR: 1.91 95\% CI: 1.10-3.32), presence of antinuclear antibodies (OR: 4.65 95\% CI: 2-12), combination therapy of methotrexate with other csDMARDs (OR: 3 95\% CI: 1.5-6), a short period of maintenance for the first medical treatment (OR: 3.32 95\% CI: 2-6 [p=0.012]), and high ESR (OR: 1.971 95\% CI: 1.1-3.5).

In multivariate analysis, three factors were independently related to the use of joint surgery: age at onset of RA (OR: 2.799 95\% CI: $1.49-5.22 ; \mathrm{p}=0.01)$, ESR (OR: 2.807 95\% CI: $1.5-5.24 ; p=0.01$ ), and combination therapy of methotrexate with other csDMARDs (OR: 3.500 95\% CI: 1.61-7.56; $\mathrm{p}=0.01$ ).

\section{DISCUSSION}

Over the last decades, the introduction of csDMARDs such as methotrexate and the acknowledgement of the importance of early diagnosis, prompt treatment, and treat-to-target approach have led to improvements in RA management. The development and introduction of bDMARDs in clinical practice and the modern treatment of RA have reduced the need for surgical treatment. ${ }^{11}$ Our study has confirmed these results and showed a reduction in surgical treatment indications.

Indeed, a maximum of orthopedic surgery procedures was noted in 2004 (30\%) and a decrease in surgical indications (4\%) was observed between 2005 and 2013 .

In our study, surgical treatment was dominated by knee and hips arthroplasty. Our results were in conformity with other international studies that showed the reduction in surgical treatment indications. ${ }^{11-13}$

In a Norwegian population, ${ }^{13}$ a study conducted in $6394 \mathrm{RA}$ patients between 1994 and 2012 showed that the incidence of arthroplasties decreased from $7.5 \mathrm{p} / 100,000$ person-year in 1997 to $0.5 / 100,000$ person-year in 2012 . Concerning synovectomy and arthrodesis, their rate decreased from 5.4 and 10.3/100,000 person-year in 1997 to 1.1 and 5.7/100,000 person-year in 2012, respectively.

Moreover, in Japanese patients with RA, ${ }^{12}$ total number of surgical treatment decreased from $72 / 1,000$ in 2004 to $51.5 / 1,000$ in 2014 with a reduction from $11.5 / 1,000$ to $6.1 / 1,000$ for total hip replacement and from $30.1 / 1,000$ to $8.51 / 1,000$ for total knee replacement.

Concerning epidemiological characteristics, no significant difference was found in our study between the two groups concerning age, sex and living environment. These results were in contrast with other studies concluding that young age at disease onset and female sex were predictive factors for surgical treatment. ${ }^{14,15}$ This difference concerning age could be explained by the fact that in our study, age was not considered as an exclusion criterion, whereas most authors included patients over 40 years of age in their studies.

In this study, no significant association was found between BMI and surgical treatment; however, BMI was not evaluable in all patients, so it was difficult to make conclusions. An American study has found a greater incidence of joint surgery use after a 10-year follow-up in obese patients (27.6\%) compared to non-obese patients 
(23.3\%) with a statistical significance $(p=0.022)$. This association was noted more frequently in knee joint surgery. ${ }^{16}$

In our study, articular deformities were associated with surgical treatment (OR: 4.16 95\% CI: 1-13.1). Esthetic reason was not a factor for surgical treatment in other studies. ${ }^{14}$ We have found that surgical treatment was associated with young age of disease onset (OR: 2.79 95\% CI: 1.49-5.22), in concordance with a Japanese study. ${ }^{12}$

Feldman et al. ${ }^{17}$ have shown that surgical treatment was more often indicated in patients who consulted tardily $(17.3 \%$ of surgical treatment in patients with time to diagnosis $<3$ months [OR: 1.09 95\% CI: 0.71-2.71] versus $23.8 \%$ in patients with time to diagnosis >3 months [OR: 1.82 95\% CI: 1.14-2.92]). Verstappen et al. ${ }^{18}$ have revealed in their study conducted on 482 RA patients that patients in whom csDMARDs were introduced immediately after diagnosis had fewer surgical indications compared to patients treated tardily. In addition, the rate of RA patients with surgical treatment was $10 \%$ lower in the group that responded effectively to medical treatment.

In our study, the duration of RA progression was associated with the use of surgery with a higher incidence of surgery in older RA patients (OR: 2.9 95\% CI: 1.1-3.6). A retrospective study of Stamp et al. ${ }^{19}$ who had followed-up RA patients for 17 years between 1999 and 2015 has shown that the duration of disease progression was longer in patients who had surgical treatment compared to patients who did not have surgical treatment $(p=0.02)$.

Concerning extra-articular manifestations, our results have joined those of Feldman et al. ${ }^{17}$ who have not demonstrated any significant association between the existence of extraarticular manifestations and the use of surgery in their study conducted in 1,051 cases of RA patients.

We have objectified a greater use of joint surgery in osteoporotic patients (OR: 1.91 95\% CI: 1.10-3.32). Our study was in conformity with the data in the literature. Indeed in 2017, a retrospective study of Mochizuki et al. ${ }^{20}$ in 57 patients followed-up for RA evolving for more than 20 years found a correlation between the presence of osteoporosis and the severity of joint erosive damage $(p<0.001)$.

Concerning laboratory investigations, our study has shown that only ESR predicted the use of joint surgery (OR: 2.807 95\% $\mathrm{CI}$ : 1.5-5.24). The evaluation of the association between ESR and CRP rate and surgical treatment has indicated divergent results across different studies. A retrospective study by Poole et al. ${ }^{21}$ in a RA population has concluded that $\mathrm{CRP}$ value predicted progression to joint replacement indication (OR: 1.36 95\% CI: 1.10-1.67). Furthermore, Kapetanovic et al. ${ }^{22}$ have studied predictive factors of orthopedic surgery use in 183 RA cases. According to them, patients who underwent surgery had higher ESR and CRP values compared to patients with no surgical indication $(p<0.001$, respectively).

In our study, the presence of RF and anti$\mathrm{CCP}$ were not associated with an increased risk of surgery during RA, whereas several studies demonstrated the association between positivity and rates of $\mathrm{RF}$ anti-CCP and severity of joint destruction in patients with RA..$^{23-25}$

We have found that the erosive character of RA in radiographic investigations did not influence the use of surgery, whereas other studies have identified early radiographic changes as early predictors of joint surgery. .11,22,26 $^{2}$

Our study has revealed that activity parameters of RA were not associated with greater surgical treatment indication. In a Japanese study, results have shown that for the 35 cases who underwent surgery, the number of painful joints was more important and the DAS28-CRP and HAQ scores were higher $(p=0.1399, p=0.0012, p=0.0001$, respectively). ${ }^{27}$ Nikiphorou et al. ${ }^{28}$ have supported these results in a retrospective study of $1,465 \mathrm{RA}$ patients and noticed that DAS28-CRP and HAQ scores were among the predictors of joint surgery ( $\mathrm{p} \leq 0.001$ and $\mathrm{p}=0.014$, respectively).

Concerning treatment, our study has demonstrated that early introduction of csDMARD was inversely associated with surgical treatment indication. Patients who responded to treatments and maintained the first treatment for a longer time had less indication for surgical treatment. 
A study of Jämsen et al. ${ }^{11}$ conducted between 1995 and 2010 to assess the impact of therapeutic progress in RA treatment in the frequency of surgical treatment need showed a reduction of two to four times in surgical treatment indications. This was concomitant to the increase in the number of patients using methotrexate (OR: 2.60 95\% CI: 2.54-2.66)

Moreover, Widdifield et al. ${ }^{29}$ followed-up RA patients for four to five years and noted that the earlier introduction of csDMARD decreased the incidence of surgery $(2-3 \%$ decrease for each month). This study has concluded that csDMARDs introduced in the shortest time after the diagnosis of RA would delay the need for surgery. All studies have converged towards the importance of aggressive csDMARDs treatment that had to be introduced rapidly from diagnosis. Similarly, delayed csDMARD introduction was a predictor factor for surgical treatment in a study of Verstappen et al. ${ }^{19}$ with OR: 1.74 95\% CI: 1.094-2.793. The nature of the csDMARDs molecules had no effect on surgical outcomes in our study as in the various studies of the literature.

The introduction of TNF antagonists and biological therapies in the late 1990s has improved the prognosis of RA and showed efficacy in stopping radiological progression and erosions. ${ }^{30,31}$ Our results were in concordance with other studies: the increase in biologic agents use was concomitant with the reduction in surgical treatment need, whereas the non-prescription of biologic agents was not a predictive factor for surgical treatment. ${ }^{32}$ These results were explained by the fact that biological treatment was administrated in cases of severe RA with significant and advanced structural damage.

Our study has indicated that the use of a combination of two csDMARDs was associated with a higher risk of surgical treatment (OR: 3.50 95\% CI: 1.61-7.56). This may be explained by the fact that patients who were treated by a combination of csDMARDs had severe RA or did not have the possibility of receiving biological treatment. Moura et al. ${ }^{33}$ have found in their study that patients who took a combination of csDMARDs had a shorter time between the onset of RA and the use of surgery compared to patients treated with one csDMARD. This study has presented several interesting points. The significant number of patients enrolled permitted statistically reliable results and the retrospective character of the study allowed to take into account the 15-year evolution of therapeutic attitudes and characteristics of RA patients.

Nevertheless, our study has some limits. Some data were missing from the files because of the retrospective nature of the study. Moreover, the difficulty in affording biological treatment in our country for some patients with RA (due to problems in health insurance or unavailability of some molecules) could compromise precise evaluation of the use of surgical treatment according to the evolution in the use of biotherapy.

In conclusion, our study has shown that surgical treatment in Tunisian RA patients was frequent (12\%). Predictive factors for surgery were age at disease onset, high ESR and association of methotrexate with other csDMARDs. Understanding these factors and their appropriate management as well as establishing early diagnosis and prompt treatment would assist in improving the overall management of RA.

\section{Declaration of conflicting interests}

The authors declared no conflicts of interest with respect to the authorship and/or publication of this article.

\section{Funding}

The authors received no financial support for the research and/or authorship of this article.

\section{REFERENCES}

1. Scott DL, Wolfe F, Huizinga TW. Rheumatoid arthritis. Lancet 2010;376:1094-108.

2. Smolen JS, Landewé R, Breedveld FC, Dougados M, Emery P, Gaujoux-Viala C, et al. EULAR recommendations for the management of rheumatoid arthritis with synthetic and biological diseasemodifying antirheumatic drugs. Ann Rheum Dis 2010;69:964-75.

3. Uhlig T, Heiberg T, Mowinckel P, Kvien TK. Rheumatoid arthritis is milder in the new millennium: health status in patients with rheumatoid arthritis 1994-2004. Ann Rheum Dis 2008;67:1710-5.

4. Hayashi M, Kojima T, Funahashi K, Kato D, Matsubara $H$, Shioura T, et al. Effect of total arthroplasty combined with anti-tumor necrosis factor agents in attenuating systemic disease activity in patients with rheumatoid arthritis. Mod Rheumatol 2012;22:363-9. 
5. Osnes-Ringen H, Kvien TK, Henriksen JE, Mowinckel P, Dagfinrud H. Orthopaedic surgery in 255 patients with inflammatory arthropathies: longitudinal effects on pain, physical function and health-related quality of life. Ann Rheum Dis 2009;68:1596-601.

6. Oh $\mathrm{K}$, Ishikawa $\mathrm{H}$, Abe $\mathrm{A}$, Otani $\mathrm{H}$, Nakazono $\mathrm{K}$, Murasawa A. Effects of surgical intervention on disease activity of rheumatoid arthritis: cases of surgery for rheumatoid arthritis of the lower limbs treated with biologics. Mod Rheumatol 2014;24:606-11.

7. Arnett FC, Edworthy SM, Bloch DA, McShane DJ, Fries JF, Cooper NS, et al. The American Rheumatism Association 1987 revised criteria for the classification of rheumatoid arthritis. Arthritis Rheum 1988;31:315-24.

8. Neogi T, Aletaha D, Silman AJ, Naden RL, Felson DT, Aggarwal R, et al. The 2010 American College of Rheumatology/European League Against Rheumatism classification criteria for rheumatoid arthritis: Phase 2 methodological report. Arthritis Rheum 2010;62:2582-91.

9. van der Heijde DM, van 't Hof M, van Riel PL, van de Putte LB. Development of a disease activity score based on judgment in clinical practice by rheumatologists. J Rheumatol 1993;20:579-81.

10. Fries JF, Spitz P, Kraines RG, Holman HR. Measurement of patient outcome in arthritis. Arthritis Rheum 1980;23:137-45.

11. Jämsen E, Virta LJ, Hakala M, Kauppi MJ, Malmivaara A, Lehto MU. The decline in joint replacement surgery in rheumatoid arthritis is associated with a concomitant increase in the intensity of anti-rheumatic therapy: a nationwide register-based study from 1995 through 2010. Acta Orthop 2013;84:331-7.

12. Matsumoto $\mathrm{T}$, Nishino J, Izawa $\mathrm{N}$, Naito $\mathrm{M}$, Hirose J, Tanaka S, et al. Trends in Treatment, Outcomes, and Incidence of Orthopedic Surgery in Patients with Rheumatoid Arthritis: An Observational Cohort Study Using the Japanese National Database of Rheumatic Diseases. J Rheumatol 2017;44:1575-82.

13. Nystad TW, Fenstad AM, Furnes O, Havelin LI, Skredderstuen AK, Fevang BT. Reduction in orthopaedic surgery in patients with rheumatoid arthritis: a Norwegian register-based study. Scand J Rheumatol 2016;45:1-7.

14. Chung KC, Kotsis SV, Kim HM, Burke FD, Wilgis EF. Reasons why rheumatoid arthritis patients seek surgical treatment for hand deformities. J Hand Surg Am 2006;31:289-94.

15. Waljee J, Zhong L, Baser O, Yuce H, Fox DA, Chung $\mathrm{KC}$. The incidence of upper and lower extremity surgery for rheumatoid arthritis among Medicare beneficiaries. J Bone Joint Surg [Am] 2015;97:403-10.

16. Shourt CA, Crowson CS, Gabriel SE, Matteson EL. Orthopedic surgery among patients with rheumatoid arthritis 1980-2007: a population-based study focused on surgery rates, sex, and mortality. J Rheumatol 2012;39:481-5.

17. Feldman DE, Bernatsky S, Houde M, Beauchamp ME, Abrahamowicz M. Early consultation with a rheumatologist for RA: does it reduce subsequent use of orthopaedic surgery? Rheumatology (Oxford) 2013;52:452-9.

18. Verstappen SM, Hoes JN, Ter Borg EJ, Bijlsma JW, Blaauw AA, van Albada-Kuipers GA, et al. Joint surgery in the Utrecht Rheumatoid Arthritis Cohort: the effect of treatment strategy. Ann Rheum Dis 2006;65:1506-11.

19. Stamp LK, Haslett J, Chapman P, O'Donnell J, Raja R, Rothwell A, et al. Rates of Joint Replacement Surgery in New Zealand, 1999-2015: A Comparison of Rheumatoid Arthritis and Osteoarthritis. J Rheumatol 2017;44:1823-7.

20. Mochizuki T, Yano K, Ikari K, Hiroshima R, Sakuma Y, Momohara S. Correlation between hand bone mineral density and joint destruction in established rheumatoid arthritis. J Orthop 2017;14:461-5.

21. Poole CD, Conway $\mathrm{P}$, Reynolds A, Currie CJ. The association between $\mathrm{C}$-reactive protein and the likelihood of progression to joint replacement in people with rheumatoid arthritis: a retrospective observational study. BMC Musculoskelet Disord 2008;9:146.

22. Kapetanovic MC, Lindquist E, Saxne T, Eberhardt $\mathrm{K}$. Orthopaedic surgery in patients with rheumatoid arthritis over 20 years: prevalence and predictive factors of large joint replacement. Ann Rheum Dis 2008;67:1412-6.

23. Suzuki T, Ikari K, Yano K, Inoue E, Toyama $Y$, Taniguchi A, et al. PADI4 and HLA-DRB1 are genetic risks for radiographic progression in RA patients, independent of ACPA status: results from the IORRA cohort study. PLoS One 2013;8:e61045.

24. Zhang L, Wang J, Zhang Q, Fu T, Yin R, Wang Z, et al. Factors associated with hand joint destruction in Chinese patients with rheumatoid arthritis. BMC Musculoskelet Disord 2017;18:211.

25. Kirino Y, Hama M, Takase-Minegishi K, Kunishita Y, Kishimoto D, Yoshimi R, et al. Predicting joint destruction in rheumatoid arthritis with power Doppler, anti-citrullinated peptide antibody, and joint swelling. Mod Rheumatol 2015;25:842-8.

26. da Silva E1, Doran MF, Crowson CS, O'Fallon WM, Matteson EL. Declining use of orthopedic surgery in patients with rheumatoid arthritis? Results of a longterm, population-based assessment. Arthritis Rheum 2003;49:216-20.

27. Sugita S, Chikuda H, Kadono Y, Ohtsu H, Takeshita K, Nishino J, et al. Clinical characteristics of rheumatoid arthritis patients undergoing cervical spine surgery: an analysis of National Database of Rheumatic Diseases in Japan. BMC Musculoskelet Disord 2014;15:203.

28. Nikiphorou E, Carpenter L, Morris S, Macgregor AJ, Dixey J, Kiely P, et al. Hand and foot surgery 
rates in rheumatoid arthritis have declined from 1986 to 2011 , but large-joint replacement rates remain unchanged: results from two UK inception cohorts. Arthritis Rheumatol 2014;66:1081-9.

29. Widdifield J, Moura CS, Wang Y, Abrahamowicz M, Paterson JM, Huang A, et al. The Longterm Effect of Early Intensive Treatment of Seniors with Rheumatoid Arthritis: A Comparison of 2 Populationbased Cohort Studies on Time to Joint Replacement Surgery. J Rheumatol 2016;43:861-8.

30. Smolen JS, Aletaha D, McInnes IB. Rheumatoid arthritis. Lancet 2016;388:2023-38.

31. Nam JL, Takase-Minegishi K, Ramiro S, Chatzidionysiou K, Smolen JS6, van der Heijde D, et al.
Efficacy of biological disease-modifying antirheumatic drugs: a systematic literature review informing the 2016 update of the EULAR recommendations for the management of rheumatoid arthritis. Ann Rheum Dis. 2017;76:1113-36.

32. Leon L, Abasolo L, Carmona L, Rodriguez-Rodriguez L, Lamas JR, et al. Orthopedic surgery in rheumatoid arthritis in the era of biologic therapy. J Rheumatol 2013;40:1850-5.

33. Moura CS, Abrahamowicz M, Beauchamp ME, Lacaille D, Wang Y, Boire G, et al. Early medication use in new-onset rheumatoid arthritis may delay joint replacement: results of a large population-based study. Arthritis Res Ther 2015;17:197. 